\title{
Betaine Treatment Attenuates Chronic Ethanol-Induced Hepatic Steatosis and Alterations to the Mitochondrial Respiratory Chain Proteome
}

\author{
Kusum K. Kharbanda, ${ }^{1,2}$ Sandra L. Todero, ${ }^{1}$ Adrienne L. King, ${ }^{3}$ Natalia A. Osna, ${ }^{2}$ \\ Benita L. McVicker, ${ }^{2}$ Dean J. Tuma, ${ }^{2}$ James L. Wisecarver, ${ }^{4}$ and Shannon M. Bailey ${ }^{3}$ \\ ${ }^{1}$ Research Service-151, Veterans Affairs Nebraska-Western Iowa Health Care System, 4101 Woolworth Avenue, Omaha, \\ NE 68105, USA \\ ${ }^{2}$ Department of Internal Medicine, University of Nebraska Medical Center, Omaha, NE 68198, USA \\ ${ }^{3}$ Department of Environmental Health Sciences, University of Alabama at Birmingham, Birmingham, AL 35294, USA \\ ${ }^{4}$ Department of Pathology and Microbiology, University of Nebraska Medical Center, Omaha, NE 68198, USA
}

Correspondence should be addressed to Kusum K. Kharbanda, kkharbanda@unmc.edu

Received 29 July 2011; Accepted 31 August 2011

Academic Editor: Laura Schrum

Copyright (๑) 2012 Kusum K. Kharbanda et al. This is an open access article distributed under the Creative Commons Attribution License, which permits unrestricted use, distribution, and reproduction in any medium, provided the original work is properly cited.

\begin{abstract}
Introduction. Mitochondrial damage and disruption in oxidative phosphorylation contributes to the pathogenesis of alcoholic liver injury. Herein, we tested the hypothesis that the hepatoprotective actions of betaine against alcoholic liver injury occur at the level of the mitochondrial proteome. Methods. Male Wister rats were pair-fed control or ethanol-containing liquid diets supplemented with or without betaine $(10 \mathrm{mg} / \mathrm{mL})$ for $4-5$ wks. Liver was examined for triglyceride accumulation, levels of methionine cycle metabolites, and alterations in mitochondrial proteins. Results. Chronic ethanol ingestion resulted in triglyceride accumulation which was attenuated in the ethanol plus betaine group. Blue native gel electrophoresis (BN-PAGE) revealed significant decreases in the content of the intact oxidative phosphorylation complexes in mitochondria from ethanol-fed animals. The alcohol-dependent loss in many of the low molecular weight oxidative phosphorylation proteins was prevented by betaine supplementation. This protection by betaine was associated with normalization of SAM : S-adenosylhomocysteine (SAH) ratios and the attenuation of the ethanol-induced increase in inducible nitric oxide synthase and nitric oxide generation in the liver. Discussion/Conclusion. In summary, betaine attenuates alcoholic steatosis and alterations to the oxidative phosphorylation system. Therefore, preservation of mitochondrial function may be another key molecular mechanism responsible for betaine hepatoprotection.
\end{abstract}

\section{Introduction}

Chronic ethanol exposure has been shown to significantly alter liver mitochondrial structural and functional integrity. Ethanol consumption alters mitochondrial morphology, induces mitochondrial DNA damage, and impairs ribosomal activity and structure [1-4] resulting in depressed mitochondrial protein synthesis and associated loss of electron transport chain complexes levels and function. It has also been shown that alcohol exposure increases the sensitivity of liver mitochondria to induce mitochondrial permeability transition pore [5] that may be linked to higher cyclophilin D levels in liver mitochondria [6]. Together, these chronic ethanol- induced alterations result in depressed respiratory capacity and impaired oxidative phosphorylation, events critical to the development of alcoholic liver injury [7-10].

In recent years, advancements in proteomic technologies have facilitated the examination of alcohol-dependent alterations to the mitochondrial proteome [11]. Using both conventional and blue native (BN)-PAGE proteomics methods, Bailey et al. have reported that ethanol exposure results in the decrease of both nuclear and mitochondrial encoded gene products of the oxidative phosphorylation system [11]. Similar defects in the mitochondrial proteome such as reductions in cytochrome $c$ oxidase subunits and mitochondrial membrane potential, have also been reported in genetically 
altered mice exhibiting deficiency in liver levels of SAM [12], buttressing the concept that SAM plays a critical role in maintaining proper mitochondrial function.

Several studies including ours have demonstrated that while the alcohol-induced decrease in hepatic SAM levels is detrimental, it is the decreased hepatocellular SAM:S-adenosylhomocysteine (SAH) ratio that adversely affects many crucial SAM-dependent methylation reactions and the ultimate generation of many hallmark features of alcoholic liver disease [13-16]. We have further shown that the addition of SAM can normalize alcohol-induced SAM:SAH ratios [17] and preserve mitochondrial respiratory capacity by maintaining the mitochondrial genome and proteome while attenuating alcohol-dependent increases in mitochondrial superoxide production $[3,18]$.

Betaine, a methyl donor and another key metabolite of the methionine cycle, has been shown to normalize hepatocellular SAM:SAH ratio, correct defective cellular methylation reactions, and prevent the alcohol-mediated steatosis, apoptosis, and accumulation of damaged proteins $[14,17$, 19-23]. Based on this, we investigated whether betaine prevents alcohol-induced changes to the mitochondrial oxidative phosphorylation system in a rat model of chronic alcohol exposure. This assessment was complemented by determinations of liver cytochrome P450 2E1 (CYP2E1) protein and activity, glutathione (GSH) levels, SAM: SAH ratios, NOS2 expression, NO generation, and triglyceride levels.

\section{Materials and Methods}

2.1. Diet Formulation. Nutritionally adequate Lieber-DeCarli control and ethanol liquid diets [24] were purchased from Dyets, Inc. (Bethlehem, Pa, USA). The ethanol diet consisted of $18 \%$ of total energy as protein, $35 \%$ as fat, $11 \%$ as carbohydrate, and $36 \%$ as ethanol. In the control diet, ethanol was replaced isocalorically with carbohydrate such that both ethanol and control rats consumed identical amounts of all nutrients except carbohydrate.

2.2. Ethanol and Betaine Feeding Procedure. Male Wistar rats (Charles River Laboratories, Wilmington, Mass, USA) weighing 180 to $200 \mathrm{~g}$ (approximately 45-48 days old) were weight-matched and divided into four groups. Group 1 was fed the control diet. Group 2 was fed the same diet as Group 1 except 1\%(w/v) betaine was added to the diet. Group 3 was fed the ethanol diet, and Group 4 was fed the ethanol diet containing $1 \%(\mathrm{w} / \mathrm{v})$ betaine. Rats in groups $1-3$ were fed the amount of diet consumed by rats in group 4. Overall, each group consisted of 8 rats fed the appropriate diet for 45 weeks. Twenty four hours before sacrifice, the total daily volume of the diet was divided with $1 / 4$ given at 8:00 am, 1/4 at 12:00 noon, and $1 / 2$ at 4:00 pm. In addition, animals were given $1 / 4$ their respective diets 60-90 minutes prior to death. This regimen was followed to minimize differences in feeding patterns that exists between the groups of rats. The care, use, and procedures performed on these rats were approved by the Institutional Animal Care and Use Committee at the Omaha Veterans Affairs Medical Center and complied with NIH guidelines.
2.3. Liver Histology and Detection of Lipid Accumulation. Formalin fixed liver tissue was processed for hematoxylineosin staining and evaluated for steatosis and inflammation. In addition, fresh frozen liver sections were fixed in $4 \% \mathrm{w} / \mathrm{v}$ paraformaldehyde in $50 \mathrm{mM}$ PIPES, $\mathrm{pH} 7.0$, and the accumulated lipid were visualized by staining with $1 \mu \mathrm{g} / \mathrm{mL}$ BODIPY 493/503 (Invitrogen, Carlsbad, Calif, USA). After incubation, slides were washed twice with PBS and mounted with "Vectashield with DAPI" (Vector laboratories, Burmingham, Calif, USA). Images were obtained with a Zeiss 510 Meta Confocal Laser Scanning Microscope using an excitation wavelength of $488 \mathrm{~nm}$ and an emission wavelength of $505 \mathrm{~nm}$.

2.4. Triglycerides. Total lipids were extracted from the liver to quantify the triglyceride mass using the triglyceride diagnostics kit (Thermo DMA kit, Thermo Electron Clinical Chemistry, Louisville, Colo, USA) as detailed in our publication [14].

2.5. Mitochondria Isolation. Pieces of fresh liver were homogenized in cold $5 \mathrm{mmol} / \mathrm{L}$ Tris $(\mathrm{pH} 7.4$ ) containing $0.25 \mathrm{~mol} / \mathrm{L}$ sucrose and $1 \mathrm{mmol} / \mathrm{L}$ EDTA, and liver mitochondria were isolated by differential centrifugation techniques [3].

2.6. Blue Native Gel Electrophoresis. Ethanol and/or betaine effects on the levels of mitochondrial proteins that comprise the oxidative phosphorylation system were assessed using BN-PAGE proteomics as detailed [3]. Image analysis on twodimensional BN-PAGE gels was performed using Quantity One software (Bio-Rad Laboratories, Hercules, Calif, USA).

2.7. SAM, SAH, and GSH Levels. The perchloric acid extract of total liver was filtered through a $0.22 \mu \mathrm{m}$ membrane filter and directly subjected to HPLC analysis for the determination of SAM, SAH, and GSH levels, as detailed [14]. GSH levels in the mitochondrial fractions were also determined.

2.8. CYP2E1 Activity. The activity was measured in liver homogenates by the formation of 4-nitrocatechol (4-NC), as previously described [25]. CYP2E1 specific activity is expressed as nmol 4-NC produced per hr per mg protein.

2.9. NOS2 Gene Expression. NOS2 gene expression was determined by real-time quantitative PCR. Total hepatic RNA was extracted, treated with DNase I (Invitrogen, Carlsbad, Calif, USA) and used to synthesize cDNA using Taqman Reverse Transcription reaction kits (Applied Biosystems, Foster City, Calif, USA). After amplification, real-time quantitative PCR was performed using an Applied Biosystems 7500 Real-Time PCR System and Taqman Assay-on-Demand gene expression assays for rat NOS 2 and $\beta$-actin (housekeeping gene) according to the manufacturer's instructions. The comparative $\mathrm{Ct}$ method was used to determine the relative concentration of the RNA transcript and the result expressed as "fold change" relative to the housekeeping gene.

2.10. Western Blotting. Immunoblots were performed by loading equal amounts of homogenate or mitochondrial 
proteins onto SDS-PAGE gels. Isolated liver mitochondria were used to detect mitochondria proteins, while total liver homogenates were used for examining inducible nitric oxide synthase (NOS2) and CYP2E1. Levels of NOS2 protein were detected using a $1: 1,000$ dilution of antibody (BD/Pharmingen, San Diego, Calif, USA). CYP2E1 protein was detected using a $1: 2,000$ dilution (Calbiochem, Gibbstown, NJ, USA). Cytochrome $c$ oxidase subunit I was detected using 1:5,000 dilution (Molecular Probes, Eugene, Ore, USA). After membranes had been incubated with the appropriate secondary antibodies, proteins were visualized using standard enhanced chemiluminescence detection methods. The intensities of immunoreactive protein bands were quantified using Quantity One software (Bio-Rad Laboratories, Hercules, Calif, USA).

2.11. Mitochondrial NO. Levels of nitrates and nitrites (the end product of nitric oxide, $\mathrm{NO}$ ) were measured in the mitochondrial fraction using the Griess reaction as detailed [26].

2.12. Statistical Analysis. Data were analyzed by ANOVA, followed by Student's Newman-Keuls post hoc test. A $P$ value $<0.05$ was regarded as statistically significant.

\section{Results}

3.1. Liver Histopathology, Triglycerides, and SAM: SAH Ratios. The histopathological evaluation of livers within each group were consistent with our previously published data [14]. Livers from the rats fed ethanol for 4-5 weeks displayed microand macrovesicular steatosis; however, no steatosis was observed in livers of rats fed the betaine-supplemented ethanol diet (Figure 1). Indeed, these livers showing similar histology as the livers of control or the betaine-supplemented control rats (Figure 1). Visualization of lipid droplets using green fluorescent BODIPY 493/503 showed considerable accumulation of neutral lipids, including esterified cholesterol in ethanol-fed rat livers (Figure 1). Minimal lipid accumulation was observed in the controls, or betaine-supplemented ethanol fed-rat livers (Figure 1). Biochemical analysis of the liver triglycerides levels corroborated the histopathology and neutral fat staining results. A significant attenuation of hepatic triglyceride content was observed in rats fed the betainesupplemented ethanol diet as compared to the rats fed ethanol alone (Figure 2).

Ethanol consumption for 4 weeks had no effect on hepatic SAM levels, but dramatically increased SAH levels [13, 14 ], resulting in a lower SAM:SAH ratio as compared with controls (Figure 3). Feeding rats a betaine-supplemented control or ethanol diet increased hepatic SAM levels 3- and 6fold, respectively (data not shown). SAH levels followed the same pattern as SAM in these two groups (data not shown). These relative changes in the levels of SAM and SAH in both betaine-supplemented groups resulted in comparable hepatic SAM:SAH ratios as in the controls. These results were similar to our previous observations [14].

3.2. Liver NOS2 and NO Generation. Chronic ethanol consumption caused induction of NOS2 both at the gene ex- pression and the protein level (Figures 4(a) and 4(b), resp.), which is in agreement with other reports [27]. As a consequence of NOS2 induction, increased levels of nitrite/nitrate, byproducts of nitric oxide metabolism, were detected in mitochondrial fractions of ethanol-fed rats (Figure 4(c)). Interestingly, the ethanol-dependent increase in hepatic NOS2 and nitrite/nitrate levels were attenuated by supplementation of the ethanol diet with betaine (Figures 4(a)$4(\mathrm{c}))$.

3.3. Liver CYP2E1 Activity and Protein Levels. Chronic ethanol consumption induced a 10-fold increase in CYP2E1 activity (Figure 5(a)). This increase in activity was also reflected by an induction in CYP2E1 protein level (Figure 5(b)). A marginal, but statistically significant, decrease $(\sim 12 \%)$ in both CYP2E1 protein and activity was observed in livers of rats fed the betaine-supplemented ethanol diet (Figures 5(a) and $5(\mathrm{~b}))$.

3.4. Mitochondrial GSH Levels. While chronic ethanol consumption decreased total liver GSH in the current study (Figure 6(a)), mitochondrial GSH was increased following chronic ethanol consumption (Figure 6(b)). Betaine treatment had no effect on the ethanol-induced decrease in total liver GSH or increased mitochondrial GSH levels observed (Figures 6(a) and 6(b)).

3.5. Assessment of Oxidative Phosphorylation Proteins. Examination of oxidative phosphorylation proteins by BN-PAGE revealed that betaine prevented an ethanol-induced loss of oxidative phosphorylation proteins. Representative one- and two-dimension BN-PAGE proteomic maps are shown in Figures $7(\mathrm{a})$ and $7(\mathrm{c})$. Similar to our previously published data [3], the two-dimensional proteomic maps revealed a loss of respiratory chain proteins following chronic ethanol exposure. This was more apparent for proteins that comprise cytochrome $c$ oxidase (i.e., complex IV) and the NADH dehydrogenase (i.e., complex I) (Figure 7(b)). Supplementation of the ethanol diet with betaine significantly attenuated the ethanol-induced loss in these mitochondrial proteins. The effects of ethanol and betaine were also reflected in Western blot analysis of complex IV. Representative data for complex IV is shown in Figure 7(d). For example, the ethanol-dependent loss in complex IV subunit I was attenuated by the inclusion of betaine in the ethanol diet.

\section{Discussion}

The role of mitochondrial dysfunction in the pathogenesis of alcoholic liver disease has long been documented by multiple laboratories $[2-4,7-10]$. Mitochondria are a recognized source of reactive oxygen and reactive nitrogen species following ethanol consumption and are also a key target of subsequent oxidative posttranslational modifications including components of the oxidative phosphorylation system [10]. Recent studies using BN-PAGE proteomics, show that chronic ethanol consumption decreases the levels of several nuclear and mitochondrial encoded proteins that comprise the individual oxidative phosphorylation complexes [3]. We have 

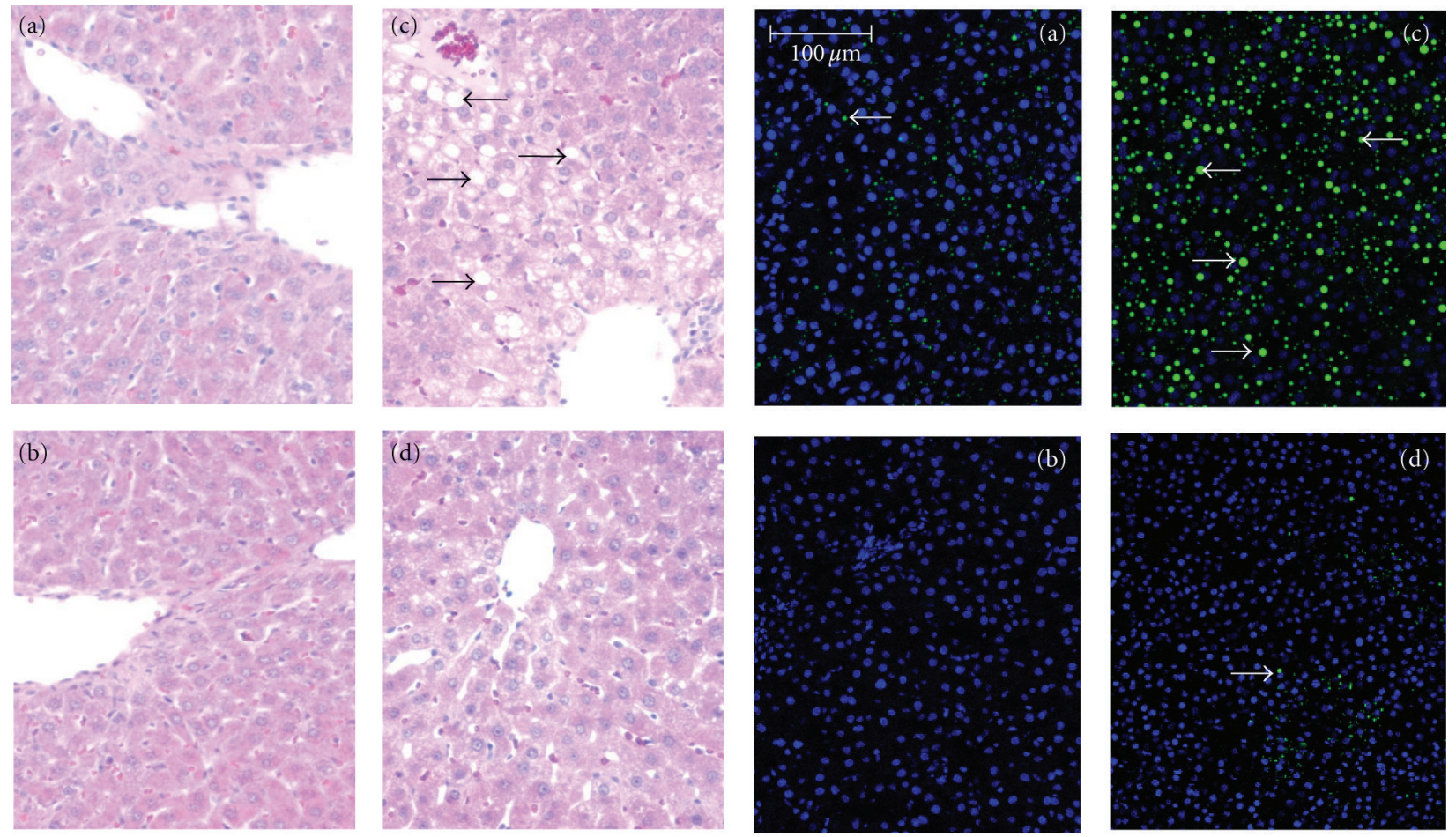

(A)

(B)

FIGURE 1: Betaine attenuates chronic ethanol-dependent steatosis. Hematoxylin-eosin (A) and BODIPY 493/503-DAPI (B, green BODIPYlabeled lipid droplets, nuclei blue) stained images from representative livers of (a) control; (b) control + betaine; (c) ethanol; (d) ethanol + betaine rats ( $n=8$ rats'group) fed the respective diets for $4-5$ weeks. Liver sections of the representative ethanol-fed rat stained with hematoxylin-eosin or BODIPY 493/503-DAPI shows micro- and macrovesicular steatosis (arrows).

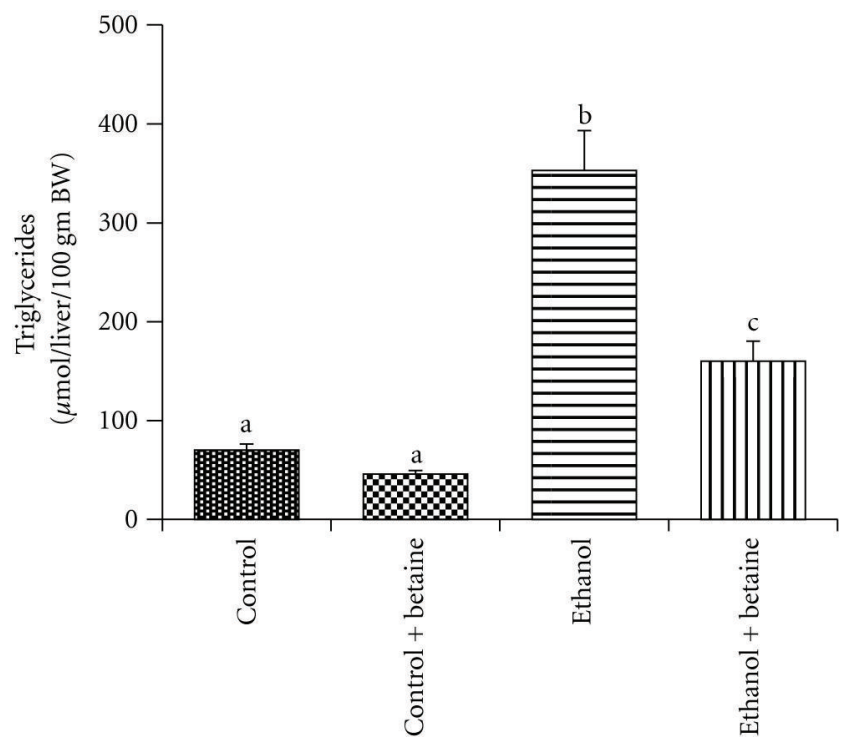

FIGURE 2: Betaine attenuates chronic ethanol-mediated increase in hepatic triglyceride levels. Triglyceride content in the liver lipid extract was quantified using the diagnostics kit (Thermo Electron Clinical Chemistry, Louisville, Colo, USA). Data represent the mean \pm S.E.M. for $n=8$ animals per treatment group. Values not sharing a common letter are statistically different, $P<0.05$.

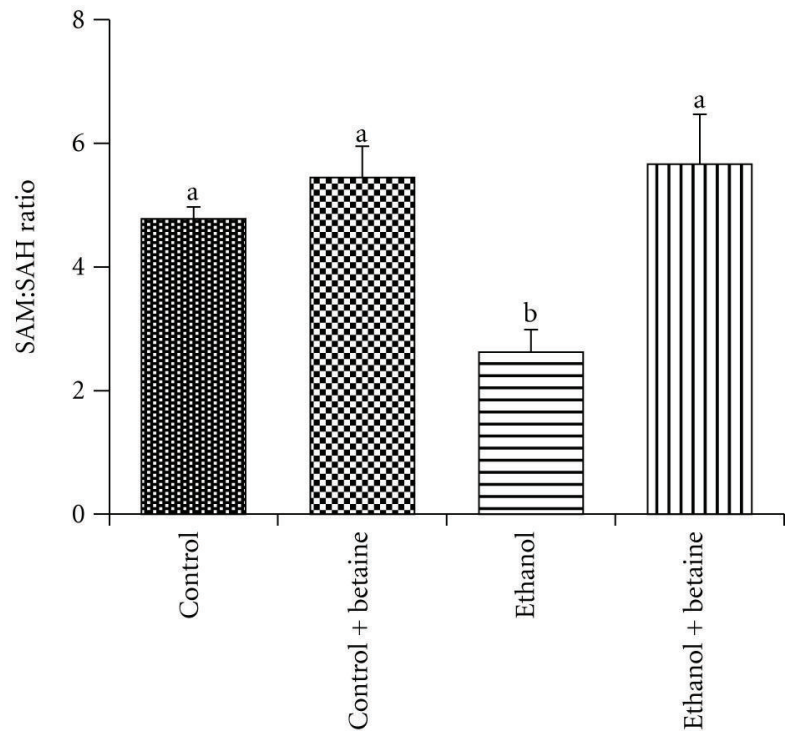

FIGURE 3: Betaine prevents the chronic ethanol-dependent decrease in the hepatic SAM:SAH ratio. Liver SAM and SAH levels were determined by HPLC analysis, and the SAM:SAH ratio was calculated as previously described [14]. Data represent the mean \pm S.E.M. for $n=8$ animals per group. Values not sharing a common letter are statistically different, $P<0.05$. 


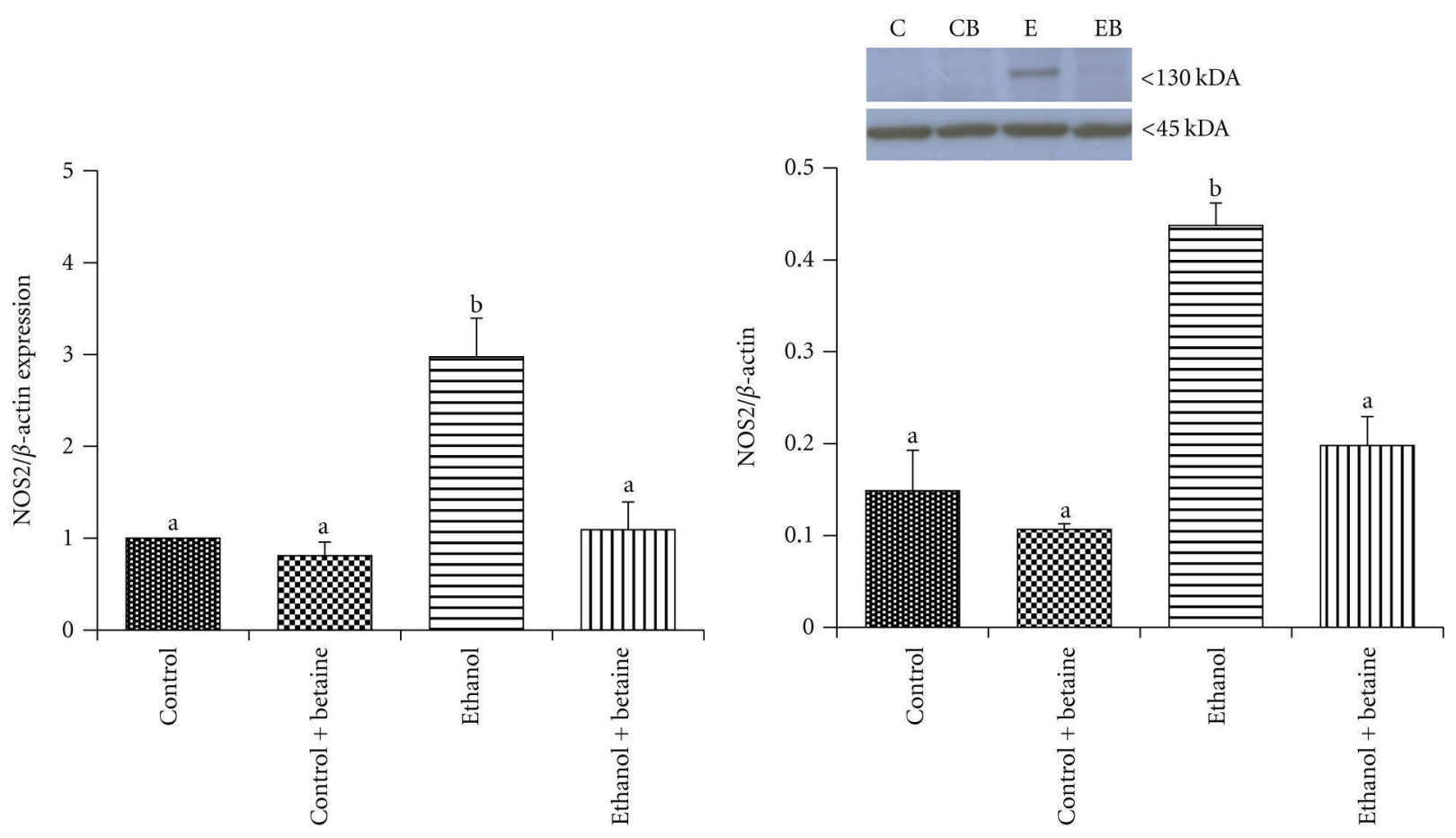

(a)

(b)

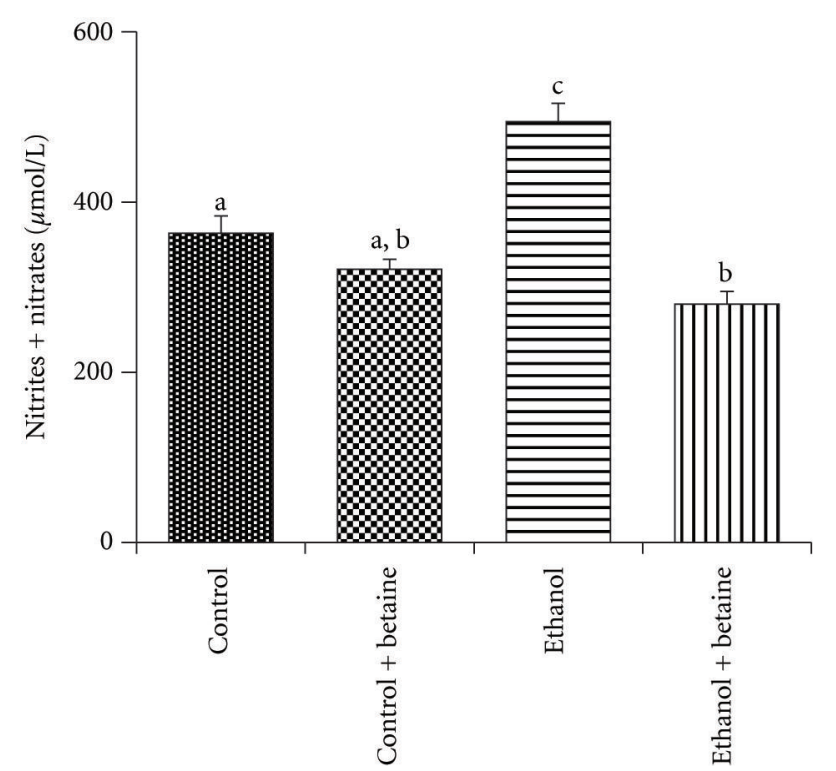

(c)

FIGURE 4: Betaine attenuates chronic ethanol-induced increase hepatic NOS2 gene and protein expression and elevates mitochondrial nitrite/nitrate levels. (a) Liver NOS2 mRNA levels were measured by quantitative PCR using the comparative Ct concentration method. The data shown are mean \pm SEM of eight determinations from each group. Values not sharing a common subscript letter are statistically different, $P<0.05$. (b) Hepatic NOS2 $(130 \mathrm{kDa})$ protein expression was measured by immunoblotting and normalized to $\beta$-actin $(45 \mathrm{kDa})$. Representative immunoblots of NOS2 protein for one pair of untreated and one pair of betaine-treated control and ethanol animals is shown. The bar graph results below represent the mean volume integration units (V.I.U) of NOS $2 \pm$ S.E.M. for $n=8$ animals per group. Values not sharing a common letter are statistically different, $P<0.05$. (c) Total mitochondrial nitrite and nitrate levels were measured by the Griess reaction as detailed [26]. The bar graph results represent the mean \pm S.E.M. for $n=8$ animals per group. Values not sharing a common letter are statistically different, $P<0.05$. 


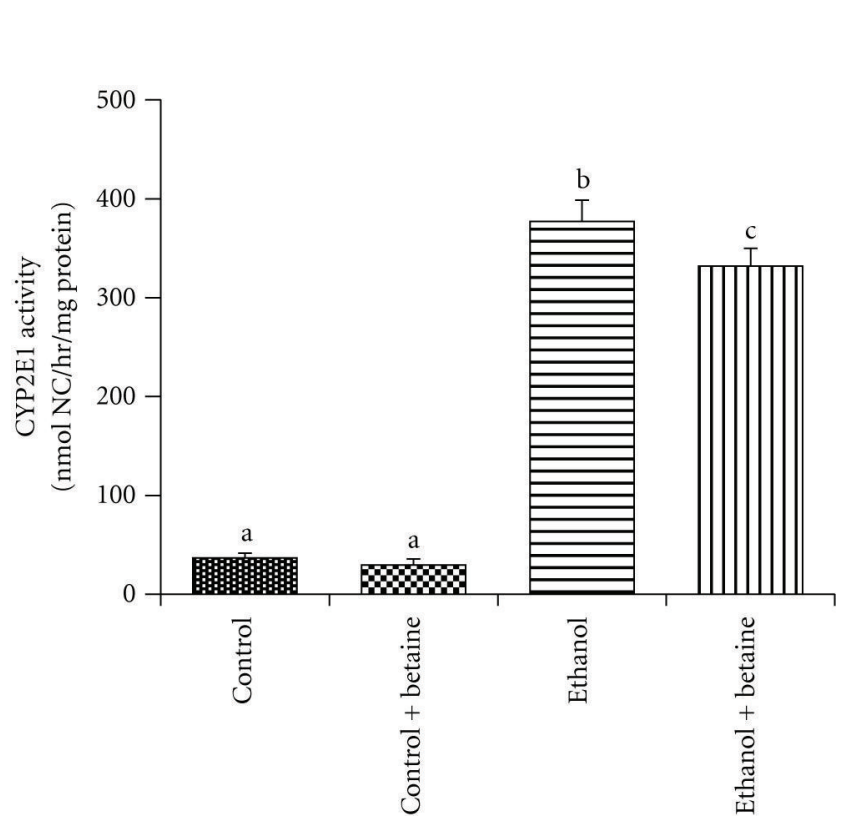

(a)

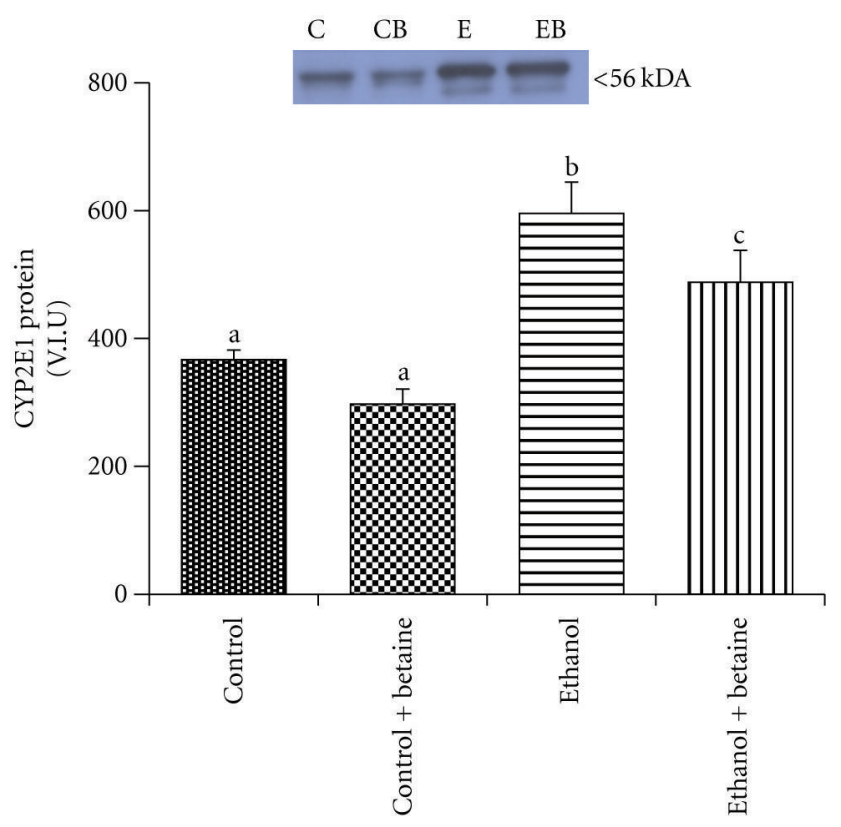

(b)

FIGURE 5: Betaine has minimal effect on chronic ethanol-dependent increase in CYP2E1 activity and protein. (a) Hepatic CYP2E1 activity was determined as detailed in Section 2. Data represent the mean \pm S.E.M. for $n=8$ animal per groups. Values not sharing a common letter are statistically different, $P<0.05$. (b) CYP2E1 protein levels were measured by immunoblotting. The bar graph results represent the mean volume integration units (V.I.U) \pm S.E.M. for $n=8$ animals per group. Values not sharing a common letter are statistically different, $P<0.05$. The top figure shows representative immunoblots of CYP2E1 protein for one pair of untreated and one pair of betaine-treated control and ethanol animals.

further shown that SAM treatment prevented these losses, particularly, select subunits of Complexes I and IV, which may explain, in part, how SAM maintains mitochondrial function and protects against the development of alcoholic liver injury $[3,18]$.

In this current study, we show for the first time that dietary supplementation with the methionine cycle metabolite, betaine, also protects against an ethanol-induced loss in oxidative phosphorylation system proteins. While the exact mechanism for this protection at the organelle level is not known, we propose that by preventing NOS2 induction and NO generation, betaine preserves the functioning of the electron transport chain, maintains the integrity of the liver, and protects against the development of alcoholic liver injury. Moreover, we propose that changes are associated with the normalization of hepatic SAM:SAH ratio and maintenance of methylation potential in response to betaine supplementation during chronic ethanol ingestion.

It is notable that NOS2 is absent in healthy, normal liver until it is transcriptionally activated by proinflammatory stimuli to produce large amounts of nitric oxide [27, 28]. Arguably, the chronic ethanol-mediated decrease in the SAM : SAH ratio in liver could be responsible for the ethanoldependent increase in NOS2 expression, since studies have shown that impaired methylation increases NOS2 gene expression and nitric oxide [29]. Thus, lower hepatocellular SAM : SAH ratios would favor hypomethylation of the NOS2 promoter leading to increased NOS2 gene transcription and subsequent NOS2 protein increase and NO generation as seen in the current study (Figure 4). Conversely, it has also been shown that hypermethylation of the NOS2 gene promoter silences and downregulates NOS2 expression in foam cells [30]. In agreement with these results, we observed that concurrent supplementation with betaine in the ethanol diet suppressed ethanol-induced NOS2 gene and protein expression and NO generation. This finding suggests that betaine through maintenance of the SAM :SAH ratio and the methylation potential in liver could prevent the upregulation of NOS2 at the level of transcription.

Studies by $\mathrm{Wu}$ and Cederbaum have proposed that CYP2E1-mediated oxidative stress causes oxidative injury to the mitochondrion [31]. It has further been suggested that the alcohol-dependent increase in CYP2E1 may be partially related to decreased proteolysis of this protein in response to impaired proteasome function in the alcoholic liver [32]. Recently, we reported that the proteasome activity is directly impaired at ratios of SAM:SAH that correspond to those observed in livers of ethanol-fed rats [33]. Indeed, these results corroborate previous observations of a negative correlation between alcohol-induced increases in liver mRNA and protein levels of CYP2E1 with SAM:SAH ratios [34]. It should also be noted that SAM interacts and inhibits the catalytic activity of CYP2E1 in a reversible and noncompetitive manner in vitro [35]. However, despite elevated SAM levels and normal SAM:SAH ratios, CYP2E1 activity was only modestly inhibited $(\sim 12 \%)$ in rats fed the betaine-supplemented ethanol diets (Figures 5(a) and 5(b)). 


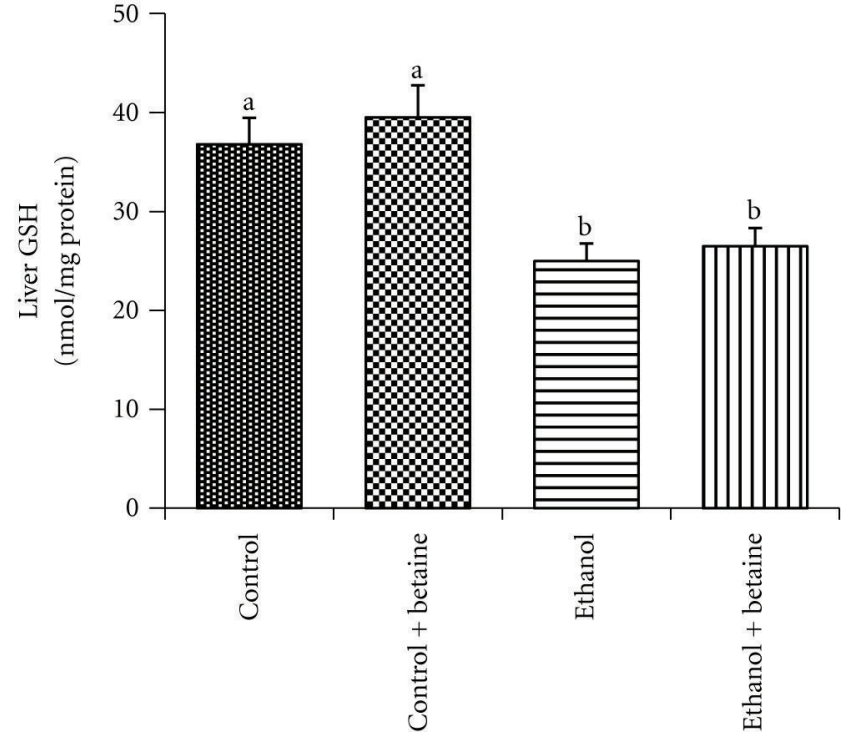

(a)

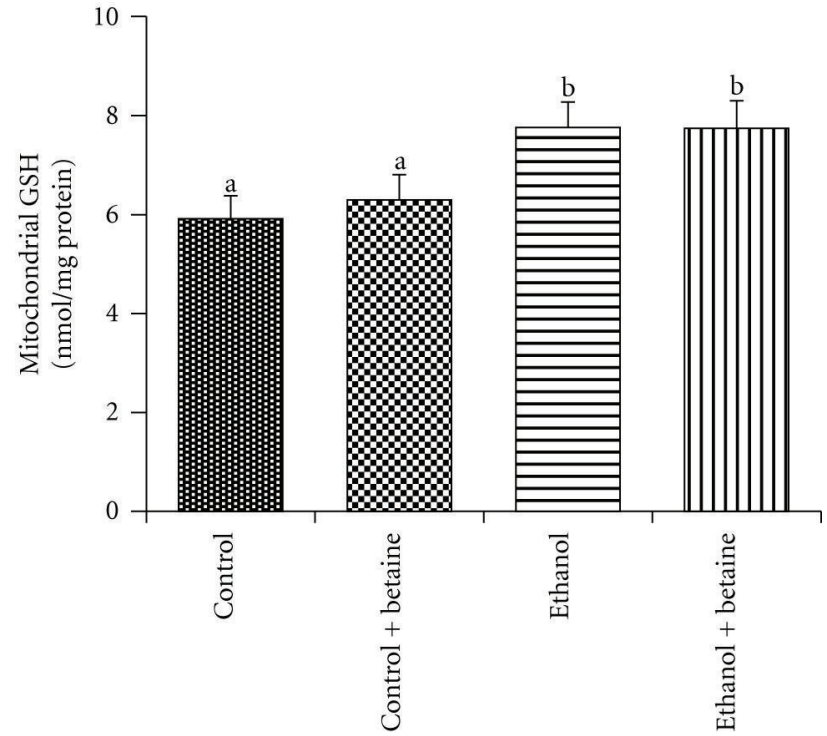

(b)

FIGURE 6: Betaine and ethanol increase mitochondrial GSH levels. HPLC was employed to determine GSH levels in (a) liver and the (b) mitochondrial fractions from control, ethanol, control + betaine, or ethanol + betaine groups. Data represent the mean \pm S.E.M. for $n=8$ animals per group. Values not sharing a common letter are statistically different, $P<0.05$.

Regardless of elevated CYP2E1 in the livers of betaine-supplemented ethanol-fed rat, indices of steatosis nor defects in the mitochondrial respiratory chain was observed in these animals $[14,17,19-23]$. Taken together, these results suggest that the increased CYP2E1 activity is not sufficient to cause and the onset of liver steatosis or mitochondrial proteome defects.

Early studies demonstrated that chronic ethanol feeding specifically caused marginal decrease in total and a marked decrease in mitochondrial GSH levels compared to controls, which was associated with mitochondrial lipid peroxidation and progression of liver damage [36, 37]. Subsequently, studies showed that feeding SAM attenuated both ethanolinduced depletion of mitochondrial GSH and mitochondrial dysfunction [38]. Contrary to these studies, an alcoholmediated increase in mitochondrial GSH has also been reported $[3,39]$. Betaine treatment has been shown to restore alcohol-induced hepatic depletion of total GSH levels [4043]. None of these studies, however, examined mitochondrial GSH status in particular following betaine treatment. In our study, we observe a significant decrease in total liver GSH (Figure 6(a)) but an (albeit small) increase in mitochondrial GSH in response to alcohol (Figure 6(b)) corroborating previous reports $[3,39]$. We further observed that betaine treatment was unable to correct alcohol-induced changes in liver or mitochondrial GSH levels (Figures 6(a) and 6(b)). Our results suggest that neither the ethanol-induced defects nor the protective role of betaine on the various parameters examined in this study appears to be mechanistically related to the status of total or mitochondrial GSH level per se.

The present data reiterates that the normal mitochondrial proteome and function relies on the maintenance of methylation reactions. Indeed, supplementation with methyl donors, SAM [3, 4], and betaine (present study) at concentrations that maintain methylation reactions preserves mitochondrial proteome $[3,4]$ as well as prevents ethanoldependent defects in mitochondrial respiration, mitochondrial ribosome dissociation, increases in mitochondrial superoxide production, and mitochondrial DNA damage $[3,4]$. While the protective action of SAM and betaine at the level of the mitochondrion is now recognized, the mechanisms responsible for this protection are not clear. Similarly, how an alteration in the SAM:SAH directly or indirectly influences the composition of the mitochondrial proteome is undefined. Recent studies by Bailey and colleagues, using a comprehensive proteomics approach, demonstrate multiple ethanol and SAM specific alterations in key proteins involved in the oxidative phosphorylation system, as well as methionine, choline, and sulfur metabolism and chaperone systems [18]. Particularly, the SAM-dependent impacts on methionine and choline metabolism enzymes may be especially important, as these effects are predicted to protect methyltransferase reactions through boosting SAM levels. Similarly, the analyses on the oxidative phosphorylation system provided further support for an association among SAM, methylation, and respiratory chain maintenance as multiple subunits of the respiratory complexes were preserved in the SAM-supplemented ethanol group [18]. These findings are further validated in the current study, as we observed the ability of betaine to attenuate the alcohol-dependent loss in complex I and IV subunits. Methylation reactions have been reported to play an important role in the biosynthesis of lipoic acid, ubiquinone and biotin [44]; key cofactors of multiple cellular and mitochondrial enzyme systems. Taken 


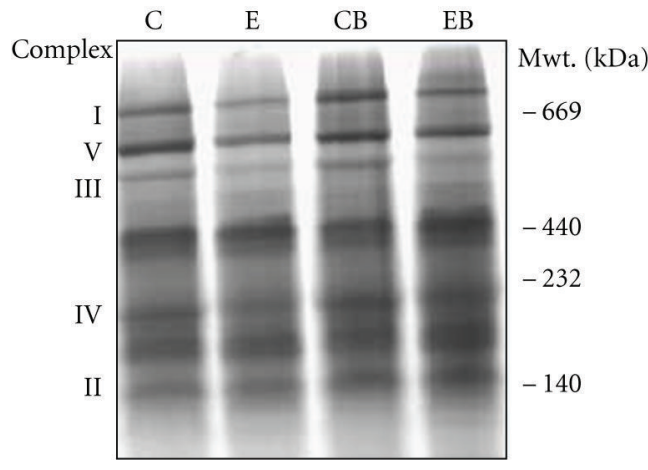

(a)

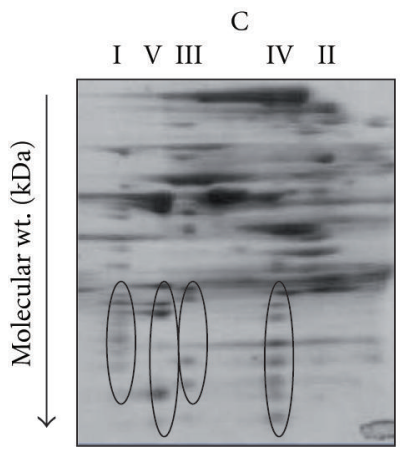

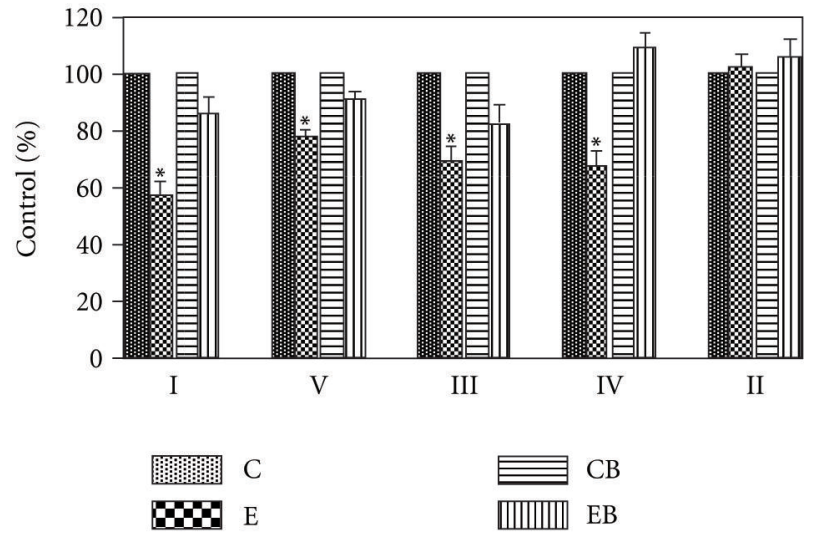

(b)
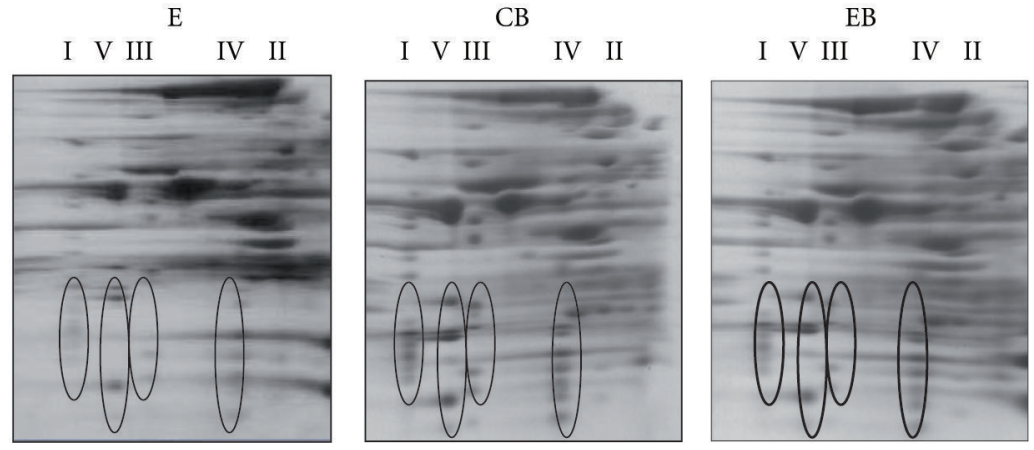

(c)

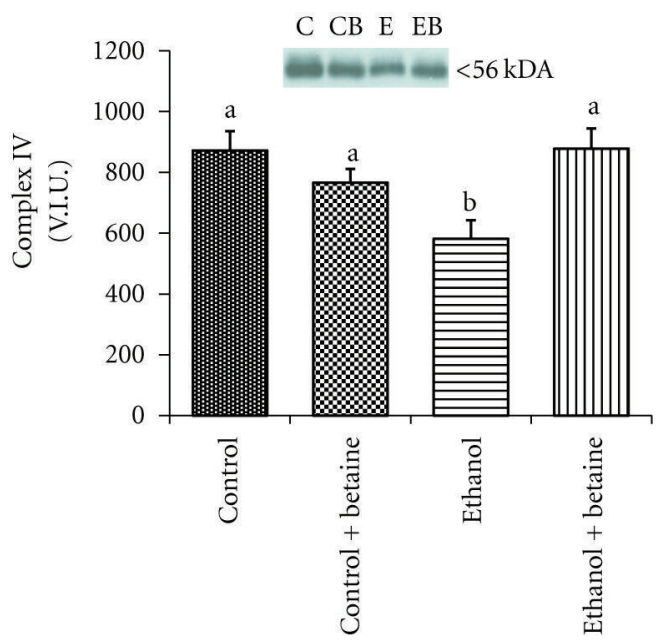

(d)

FIGURE 7: Betaine prevents the chronic ethanol-mediated loss in oxidative phosphorylation proteins. Representative (a) one- and (c) twodimension blue native (BN)-PAGE proteomic gels of mitochondria isolated from rats fed control, ethanol, control + betaine, or ethanol + betaine diets as detailed in Section 2. (a) For these gels, $250 \mu \mathrm{g}$ of mitochondrial protein were subjected to 1D BN-PAGE. (c) The 1D gel strips were overlaid across a Tris-Tricine-SDS-PAGE gel to resolve the individual polypeptides that comprise each oxidative phosphorylation system complex. The proteins that comprise each complex (I, V, III, IV, and II) appear as vertically aligned spots on the $2 \mathrm{D}$ gel. (b) Comparison of the relative quantities of complexes I, V, III, and IV in liver mitochondria from the control; control + betaine; ethanol, and ethanol + betaine. Statistical analyses for data presented in panel B: 2-factor ANOVA on raw densitometry values-complex I: ethanol $P=0.0002$, betaine $P=0.92$, interaction $P=0.02$; complex V: ethanol $P=0.016$, betaine $P=0.72$, interaction $P=0.23$; complex III: ethanol $P=0.005$, betaine $P=0.92$, interaction $P=0.31$; complex IV: ethanol $P=0.15$, betaine $P=0.62$, interaction $P=0.045$; complex II: ethanol $P=0.54$, betaine $P=0.98$, interaction $P=0.80$. (d) Complex IV, subunit 1 protein levels were measured by immunoblotting. The bar graph results represent the mean volume integration units (V.I.U) \pm S.E.M. for $n=8$ animals per group. Values not sharing a common letter are statistically different, $P<0.05$. The top figure shows representative immunoblots of subunit 1 protein for control, ethanol, and betaine supplemented control and ethanol-fed rats. 
together, future experiments will be directed at determining whether alcohol-dependent disruption in the activity of specific mitochondrial SAM-dependent methyltransferase(s) as a consequence of low SAM : SAH ratio is directly responsible for alterations in mitochondrion proteome and function.

In conclusion, this study shows for the first time that betaine prevents or blunts chronic ethanol-mediated alterations to the oxidative phosphorylation system proteome. We propose that the mitochondrial protection afforded by betaine is associated with the maintenance of hepatic SAM : SAH ratios and by blocking the ethanol-induced induction of NOS2 and NO, a key source of protein modification and damage. Moreover, this study demonstrates that proteomic-based methods like BN-PAGE are powerful tools to aid in the identification in the molecular targets of disease. Thus, maintenance of mitochondria function may be another key molecular target underlying the hepatoprotective effect of betaine against ethanol hepatotoxicity and fatty liver disease.

\section{Abbreviations}

BN-PAGE: Blue native gel electrophoresis

CYP2E1: Cytochrome P450 2E1

GSH: Glutathione

NOS2: Inducible nitric oxide synthase

SAM: S-adenosylmethionine

SAH: $\quad$ S-adenosylhomocysteine.

\section{Conflict of Interests}

The authors of this study confirm that they have no commercial associations that pose a conflict of interests in connection with this paper.

\section{Acknowledgments}

The authors gratefully acknowledge the financial support of the following: Department of Veterans Affairs National Merit Review grant (K. K. Kharbanda) and National Institute of Health grants, R21AA017296 (K. K. Kharbanda) and AA15172 and AA18841 (S. M. Bailey). Ms. King is supported by a NIH Research Supplement to Promote Diversity in Health-Related Research linked to parent grant AA15172.

\section{References}

[1] T. F. Taraschi, W. S. Thayer, J. S. Ellingson, and E. Rubin, "Effects of ethanol on the structure and function of rat liver mitochondrial and microsomal membranes," Annals of the New York Academy of Sciences, vol. 488, pp. 127-139, 1986.

[2] A. Cahill, X. Wang, and J. B. Hoek, "Increased oxidative damage to mitochondrial DNA following chronic ethanol consumption," Biochemical and Biophysical Research Communications, vol. 235, no. 2, pp. 286-290, 1997.

[3] S. M. Bailey, G. Robinson, A. Pinner et al., "S-adenosylmethionine prevents chronic alcohol-induced mitochondrial dysfunction in the rat liver," American Journal of Physiology, vol. 291, no. 5, pp. G857-G867, 2006.

[4] P. Sykora, K. K. Kharbanda, S. E. Crumm, and A. Cahill, "S-adenosyl-L-methionine co-administration prevents the ethanol-elicited dissociation of hepatic mitochondrial ribosomes in male rats," Alcoholism: Clinical and Experimental Research, vol. 33, no. 1, pp. 1-9, 2009.

[5] M. Yan, P. Zhu, H. M. Liu, H. T. Zhang, and L. Liu, "Ethanol induced mitochondria injury and permeability transition pore opening: role of mitochondria in alcoholic liver disease," World Journal of Gastroenterology, vol. 13, no. 16, pp. 23522356, 2007.

[6] A. L. King, T. M. Swain, D. A. Dickinson, M. J. Lesort, and S. M. Bailey, "Chronic ethanol consumption enhances sensitivity to $\mathrm{Ca}^{2+}$-mediated opening of the mitochondrial permeability transition pore and increases cyclophilin D in liver," American Journal of Physiology, vol. 299, no. 4, pp. G954-G966, 2010.

[7] C. C. Cunningham and S. M. Bailey, "Ethanol consumption and liver mitochondria function," Biological Signals and Receptors, vol. 10, no. 3-4, pp. 271-282, 2001.

[8] S. M. Bailey and C. C. Cunningham, "Contribution of mitochondria to oxidative stress associated with alcoholic liver disease," Free Radical Biology and Medicine, vol. 32, no. 1, pp. 1116, 2002.

[9] J. B. Hoek, A. Cahill, and J. G. Pastorino, "Alcohol and mitochondria: a dysfunctional relationship," Gastroenterology, vol. 122, no. 7, pp. 2049-2063, 2002.

[10] S. M. Bailey, "A review of the role of reactive oxygen and nitrogen species in alcohol-induced mitochondrial dysfunction," Free Radical Research, vol. 37, no. 6, pp. 585-596, 2003.

[11] S. M. Bailey, A. Landar, and V. Darley-Usmar, "Mitochondrial proteomics in free radical research," Free Radical Biology and Medicine, vol. 38, no. 2, pp. 175-188, 2005.

[12] M. L. Martínez-Chantar, F. J. Corrales, L. A. Martínez-Cruz et al., "Spontaneous oxidative stress and liver tumors in mice lacking methionine adenosyltransferase 1A," The FASEB Journal, vol. 16, no. 10, pp. 1292-1294, 2002.

[13] A. J. Barak, H. C. Beckenhauer, M. E. Mailliard, K. K. Kharbanda, and D. J. Tuma, "Betaine lowers elevated S-adenosylhomocysteine levels in hepatocytes from ethanol-fed rats," Journal of Nutrition, vol. 133, no. 9, pp. 2845-2848, 2003.

[14] K. K. Kharbanda, M. E. Mailliard, C. R. Baldwin, H. C. Beckenhauer, M. F. Sorrell, and D. J. Tuma, "Betaine attenuates alcoholic steatosis by restoring phosphatidylcholine generation via the phosphatidylethanolamine methyltransferase pathway," Journal of Hepatology, vol. 46, no. 2, pp. 314-321, 2007.

[15] K. K. Kharbanda, "Alcoholic liver disease and methionine metabolism," Seminars in Liver Disease, vol. 29, no. 2, pp. 155$165,2009$.

[16] K. K. Kharbanda, "Role of transmethylation reactions in alcoholic liver disease," World Journal of Gastroenterology, vol. 13, no. 37, pp. 4947-4954, 2007.

[17] K. K. Kharbanda, D. D. Rogers, M. E. Mailliard et al., "A comparison of the effects of betaine and S-adenosylmethionine on ethanol-induced changes in methionine metabolism and steatosis in rat hepatocytes," Journal of Nutrition, vol. 135, no. 3, pp. 519-524, 2005.

[18] K. K. Andringa, A. L. King, H. B. Eccleston et al., "Analysis of the liver mitochondrial proteome in response to ethanol and S-adenosylmethionine treatments: novel molecular targets of disease and hepatoprotection," American Journal of Physiology, vol. 298, no. 5, pp. G732-G745, 2010.

[19] C. Ji and N. Kaplowitz, "Betaine decreases hyperhomocysteinemia, endoplasmic reticulum stress, and liver injury in alcohol-fed mice," Gastroenterology, vol. 124, no. 5, pp. 14881499, 2003. 
[20] K. K. Kharbanda, D. D. Rogers II, H. C. Beckenhauer et al., "Adenosine-induced apoptosis mediated via increased hepatocellular levels of S-adenosylhomocysteine is attenuated by betaine administration," Hepatology, vol. 40, p. 572A, 2004.

[21] K. K. Kharbanda and A. J. Barak, "Defects in methionine metabolism: its role in ethanol-induced liver injury," in Comprehensive Handbook of Alcohol-Related Pathology, V. R. Preedy and R. R. Watson, Eds., vol. 2, pp. 735-747, Academic Press, Elsevier Sciences, 2005.

[22] K. K. Kharbanda, D. D. Rogers, M. E. Mailliard et al., "Role of elevated S-adenosylhomocysteine in rat hepatocyte apoptosis: protection by betaine," Biochemical Pharmacology, vol. 70, no. 12, pp. 1883-1890, 2005.

[23] K. K. Kharbanda, M. E. Mailliard, C. R. Baldwin, M. F. Sorrell, and D. J. Tuma, "Accumulation of proteins bearing atypical isoaspartyl residues in livers of alcohol-fed rats is prevented by betaine administration: effects on protein-l-isoaspartyl methyltransferase activity," Journal of Hepatology, vol. 46, no. 6, pp. 1119-1125, 2007.

[24] C. S. Lieber and L. M. Decarli, "Liquid diet technique of ethanol administration: 1989 update," Alcohol and Alcoholism, vol. 24, no. 3, pp. 197-211, 1989.

[25] N. A. Osna, D. L. Clemens, and T. M. Donohue, "Interferon gamma enhances proteasome activity in recombinant Hep G2 cells that express cytochrome P4502E1: modulation by ethanol," Biochemical Pharmacology, vol. 66, no. 5, pp. 697-710, 2003.

[26] H. Moshage, B. Kok, J. R. Huizenga, and P. L. M. Jansen, "Nitrite and nitrate determinations in plasma: a critical evaluation," Clinical Chemistry, vol. 41, no. 6, pp. 892-896, 1995.

[27] A. Venkatraman, S. Shiva, A. Wigley et al., "The role of iNOS in alcohol-dependent hepatotoxicity and mitochondrial dysfunction in mice," Hepatology, vol. 40, no. 3, pp. 565-573, 2004.

[28] S. E. McKim, E. Gäbele, F. Isayama et al., "Inducible nitric oxide synthase is required in alcohol-induced liver injury: studies with knockout mice," Gastroenterology, vol. 125, no. 6, pp. 1834-1844, 2003.

[29] Z. Yu and B. C. Kone, "Hypermethylation of the inducible nitric-oxide synthase gene promoter inhibits its transcription," The Journal of Biological Chemistry, vol. 279, no. 45, pp. 46954-46961, 2004.

[30] Y. Jiang, J. Zhang, J. Xiong, J. Cao, G. Li, and S. Wang, "Ligands of peroxisome proliferator-activated receptor inhibit homocysteine induced DNA methylation of inducible nitric oxide synthase gene," Acta Biochimica et Biophysica Sinica, vol. 39, no. 5, pp. 366-376, 2007.

[31] D. Wu and A. I. Cederbaum, "Oxidative stress and alcoholic liver disease," Seminars in Liver Disease, vol. 29, no. 2, pp. 141154, 2009.

[32] B. J. Roberts, B. J. Song, Y. Soh, S. S. Park, and S. E. Shoaf, "Ethanol induces CYP2E1 by protein stabilization: role of ubiquitin conjugation in the rapid degradation of CYP2E1," The Journal of Biological Chemistry, vol. 270, no. 50, pp. 29632-29635, 1995.

[33] N. A. Osna, R. L. White, T. M. Donohue, M. R. Beard, D. J. Tuma, and K. K. Kharbanda, "Impaired methylation as a novel mechanism for proteasome suppression in liver cells," Biochemical and Biophysical Research Communications, vol. 391, no. 2, pp. 1291-1296, 2010.

[34] F. Esfandiari, J. A. Villanueva, D. H. Wong, S. W. French, and C. H. Halsted, "Chronic ethanol feeding and folate deficiency activate hepatic endoplasmic reticulum stress pathway in mi- cropigs," American Journal of Physiology, vol. 289, no. 1, pp. G54-G63, 2005.

[35] A. A. Caro and A. I. Cederbaum, "Inhibition of CYP2E1 catalytic activity in vitro by S-adenosyl-L-methionine," Biochemical Pharmacology, vol. 69, no. 7, pp. 1081-1093, 2005.

[36] T. Hirano, N. Kaplowitz, H. Tsukamoto, S. Kamimura, and J. C. Fernandez-Checa, "Hepatic mitochondrial glutathione depletion and progression of experimental alcoholic liver disease in rats," Hepatology, vol. 16, no. 6, pp. 1423-1427, 1992.

[37] J. C. Fernandez-Checa, M. Ookhtens, and N. Kaplowitz, "Effect of chronic ethanol feeding on rat hepatocytic glutathione. Compartmentation, efflux, and response to incubation with ethanol," Journal of Clinical Investigation, vol. 80, no. 1, pp. 57-62, 1987.

[38] C. Garcia-Ruiz, A. Morales, A. Colell et al., "Feeding S-adenosyl-L-methionine attenuates both ethanol-induced depletion of mitochondrial glutathione and mitochondrial dysfunction in periportal and perivenous rat hepatocytes," Hepatology, vol. 21, no. 1, pp. 207-214, 1995.

[39] S. M. Bailey, V. B. Patel, T. A. Young, K. Asayama, and C. C. Cunningham, "Chronic ethanol consumption alters the glutathione/glutathione peroxidase- 1 system and protein oxidation status in rat liver," Alcoholism: Clinical and Experimental Research, vol. 25, no. 5, pp. 726-733, 2001.

[40] G. Kanbak, M. Inal, and C. Bayçu, "Ethanol-induced hepatotoxicity and protective effect of betaine," Cell Biochemistry and Function, vol. 19, no. 4, pp. 281-285, 2001.

[41] S. H. Kenyon, C. J. Waterfield, D. S. Asker et al., "Effect of hydrazine upon vitamin B12-dependent methionine synthase activity and the sulphur amino acid pathway in isolated rat hepatocytes," Biochemical Pharmacology, vol. 57, no. 11, pp. 1311-1319, 1999.

[42] J. Balkan, S. Öztezcan, M. Küçük, U. Çevikbaş, N. KoçakToker, and M. Uysal, "The effect of betaine treatment on triglyceride levels and oxidative stress in the liver of ethanoltreated guinea pigs," Experimental and Toxicologic Pathology, vol. 55, no. 6, pp. 505-509, 2004.

[43] C. L. Powell, B. U. Bradford, C. P. Craig et al., "Mechanism for prevention of alcohol-induced liver injury by dietary methyl donors," Toxicological Sciences, vol. 115, no. 1, pp. 131-139, 2010.

[44] G. S. Shadel, "Coupling the mitochondrial transcription machinery to human disease," Trends in Genetics, vol. 20, no. 10, pp. 513-519, 2004. 


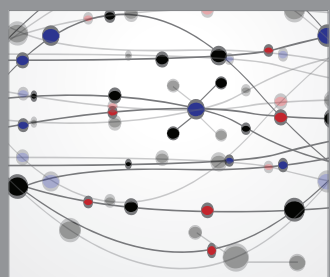

The Scientific World Journal
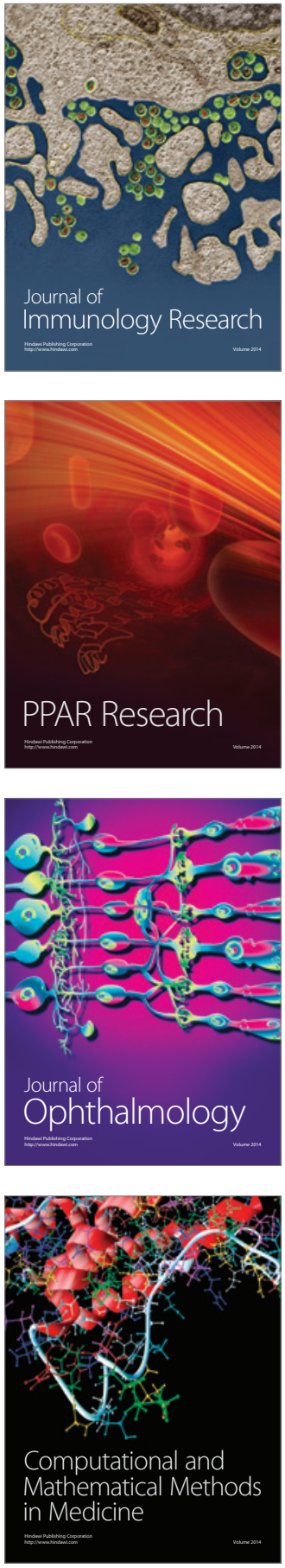

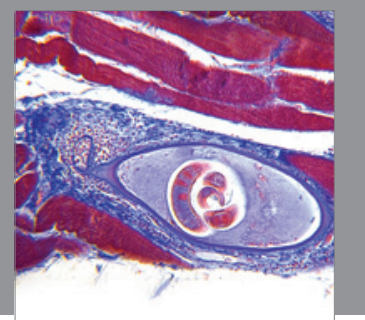

Gastroenterology

Research and Practice
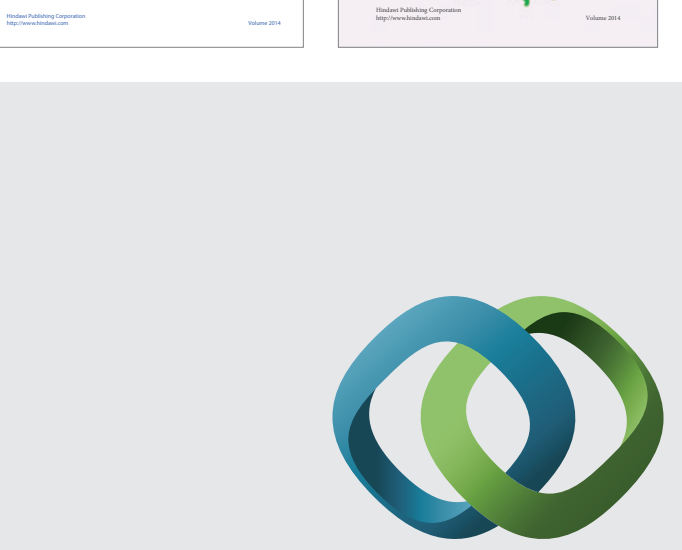

\section{Hindawi}

Submit your manuscripts at

http://www.hindawi.com
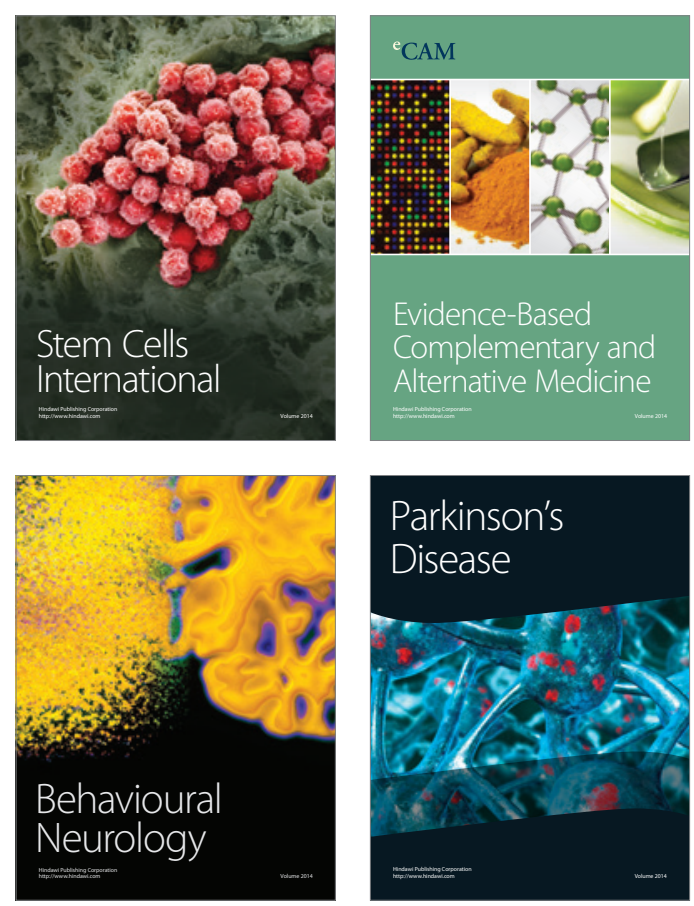

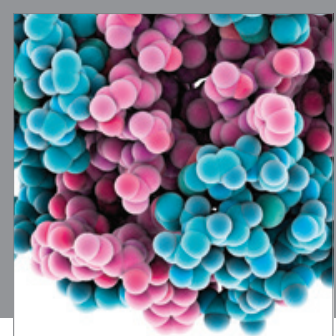

Journal of
Diabetes Research

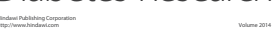

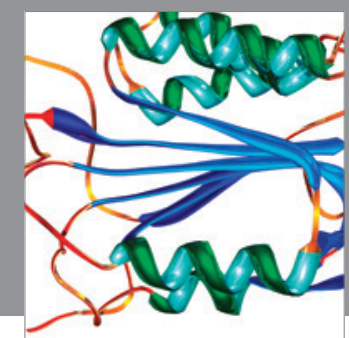

Disease Markers
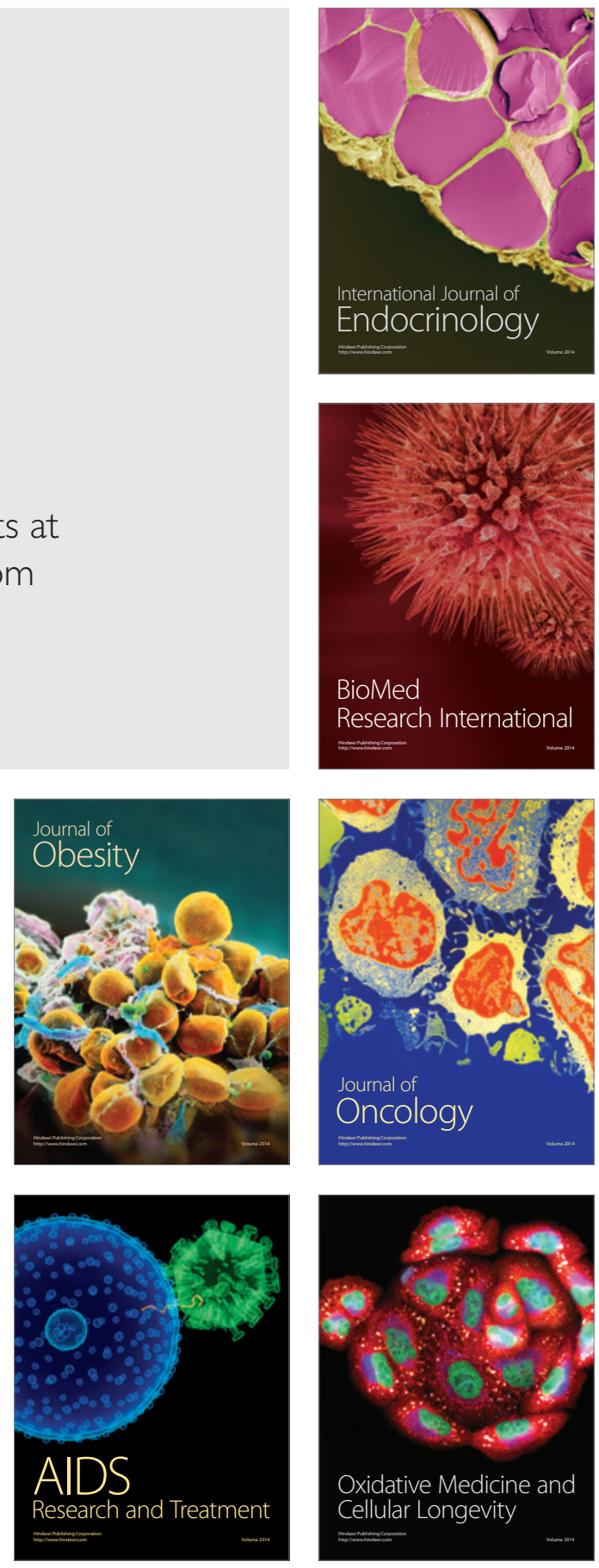